\title{
Development and Fabrication of Advanced Materials for Energy and Environment Applications 2014
}

\author{
Ming-Guo Ma, ${ }^{1}$ Wen Zeng, ${ }^{2}$ Jie-Fang Zhu, ${ }^{3}$ Shao-Wen Cao, ${ }^{4}$ and Zhong-Chang Wang ${ }^{5}$ \\ ${ }^{1}$ Beijing Key Laboratory of Lignocellulosic Chemistry, College of Materials Science and Technology, Beijing Forestry University, \\ Beijing 100083, China \\ ${ }^{2}$ College of Materials Science and Engineering, Chongqing University, Chongqing 400040, China \\ ${ }^{3}$ Department of Materials Chemistry, The Angström Laboratory, Uppsala University, 75121 Uppsala, Sweden \\ ${ }^{4}$ School of Materials Science and Engineering, Nanyang Technological University, Singapore 639798 \\ ${ }^{5}$ WPI Research Center, Advanced Institute for Materials Research, Tohoku University, Sendai 980-8577, Japan \\ Correspondence should be addressed to Ming-Guo Ma; mg_ma@bjfu.edu.cn
}

Received 5 June 2014; Accepted 5 June 2014; Published 19 June 2014

Copyright (c) 2014 Ming-Guo Ma et al. This is an open access article distributed under the Creative Commons Attribution License, which permits unrestricted use, distribution, and reproduction in any medium, provided the original work is properly cited.

Recently, environmental and energy problems have been receiving more and more attention. It is reported that advanced materials are promising applications in environmental and energy fields. Therefore, this special issue focused on the development and fabrication of advanced materials for energy and environment applications. It is expected that this special issue would provide a communication platform on the recent development of these fields.

The Call for Papers of the special issue was posted online on November 10, 2013, and closed for submission on February 21,2014 . We received 24 papers for submission in less than four months. Finally, 19 papers were selected for publication.

J. C. Wang et al. applied the hydrothermal method for the synthesis of the Cu/SAPO-34 catalysts with different $\mathrm{Cu}$ contents, which was modified by impregnating Pd. It was found that the $\mathrm{Cu} / \mathrm{SAPO}-34$ catalysts with $\mathrm{Cu} / \mathrm{Si}$ ratios of $0.05,0.1$, and 0.2 had better de- $\mathrm{NO}_{x}$ activity than other catalysts, by the selective catalytic reduction by $\mathrm{C}_{3} \mathrm{H}_{6}$ and $\mathrm{NH}_{3}$. Experimental results indicated that the maximum conversion of NO with Pd could reach $90 \%$.

L. Zeng et al. reported the synthesis and characterization of calcium silicate hydrates by hydrothermal method, which was used to adsorb carcinogenic aflatoxin B1. It was shown that the adsorption followed the pseudosecond-order kinetic model and the data of equilibrium adsorption were fitted to Langmuir and Freundlich equations.

The mechanism especially for $\mathrm{H}_{2}$ molecules adsorption on solid $\mathrm{C}_{60}$ under high pressure was proposed by $\mathrm{H}$. Wang et al. via a first principle comparative study. They demonstrated that the initial $\mathrm{H}_{2}$ adsorption on two adjacent $\mathrm{C}_{60}$ gave a much lower barrier $1.36 \mathrm{eV}$ in comparison with the barrier of adsorption on a single $\mathrm{C}_{60}$ (about $3.0 \mathrm{eV}$ ).

$\mathrm{S}$. Zhang et al. prepared the organic/inorganic superabsorbent hydrogels based on xylan and inorganic clay montmorillonite via grafting copolymerization of acrylic acid and 2-acrylamido-2-methylpropanesulfonic acid with $\mathrm{N}, \mathrm{N}$ methylenebisacrylamide as a cross-linking agent and potassium persulfate as an initiator. Experimental results indicated that the hydrogels exhibited the high compressive modulus about 35-55 KPa. The maximum equilibrium swelling ratios of hydrogels in distilled water and $0.9 \mathrm{wt} \%$ sodium chloride solutions were up to $1423 \mathrm{~g} \mathrm{~g}^{-1}$ and $69 \mathrm{~g} \mathrm{~g}^{-1}$, respectively. The effect of various cationic salt solutions $\left(\mathrm{LiCl}, \mathrm{CaCl}_{2}\right.$, and $\mathrm{FeCl}_{3}$ ) on the swelling has the following order: $\mathrm{Li}^{+}>\mathrm{Ca}^{2+}>$ $\mathrm{Fe}^{3+}$.

X. Yu et al. prepared urea-formaldehyde paraffin capsules modified by $\beta$-cyclodextrin with excellent energy storage capacity using different emulsifiers. It was reported that OP10 for use with PCMs as emulsifier had a better emulsifying 
capacity, thermal stability, and mechanical stirring stability than SDBS for use with PCMs as emulsifier.

J. Wang et al. synthesized $\mathrm{Mn} / \mathrm{Si}$ complexes in supercritical water using a tube reactor by a series of manganese salts $\left(\mathrm{Mn}\left(\mathrm{NO}_{3}\right)_{2}, \mathrm{MnCl}_{2}, \mathrm{MnSO}_{4}\right.$, and $\left.\mathrm{Mn}(\mathrm{Ac})_{2}\right)$ and silicon materials (silica sand, silica sol, and tetraethyl orthosilicate). It was found that $\mathrm{MnO}_{2}, \mathrm{Mn}_{2} \mathrm{O}_{3}$, and $\mathrm{Mn}_{2} \mathrm{SiO}_{4}$ could be obtained in supercritical water at $673 \mathrm{~K}$ in $5 \mathrm{~min}$ and the hydroxyl groups on the surface of $\mathrm{SiO}_{2}$ from different silicon sources enhanced the reactivity of $\mathrm{SiO}_{2}$. H. S. Hassan et al. prepared zinc oxide with $94.9 \%$ dye removal ability within $1 \mathrm{~h}$ by sol-gel technique, which was a promising adsorbent material for dye decolorization from the polluted water.

As promising hydrogen storage materials, ammonia borane $\left(\mathrm{NH}_{3} \mathrm{BH}_{3}\right)$ and hydrazine borane $\left(\mathrm{N}_{2} \mathrm{H}_{4} \mathrm{BH}_{3}\right)$ have hydrogen content as high as $19.6 \mathrm{wt} \%$ and $15.4 \mathrm{wt} \%$. Z.-H. $\mathrm{Lu}$ et al. survey the research progresses in nanocatalysts for hydrogen generation from the hydrolysis or methanolysis of $\mathrm{NH}_{3} \mathrm{BH}_{3}$ and $\mathrm{N}_{2} \mathrm{H}_{4} \mathrm{BH}_{3}$. Moreover, they synthesized well dispersed magnetically recyclable bimetallic $\mathrm{CoNi}$ nanoparticles supported on the reduced graphene oxide by one-step in situ coreduction of aqueous solution of cobalt(II) chloride, nickel(II) chloride, and graphite oxide with ammonia borane as the reducing agent under ambient condition. It was found that the $\mathrm{CoNi} /$ reduced graphite oxide nanoparticles exhibited excellent catalytic activity with a total turnover frequency value of $19.54 \mathrm{~mol} \mathrm{H}_{2}$ mol catalyt-1 $\mathrm{min}^{-1}$ and a low activation energy value of $39.89 \mathrm{~kJ} \mathrm{~mol}^{-1}$ at room temperature.

C.-d. Gao et al. prepared miscible, biodegradable poly(vinyl alcohol)/xylan blending films in the range of the PVA/xylan weight ratio from $1: 2$ to $3: 1$ by casting method using 1,2,3,4-butane tetracarboxylic acid as a new plasticizer. It was shown that blending films were biodegraded almost by $41 \%$ with an addition of $10 \%$ 1,2,3,4-butane tetracarboxylic acid in blending films within 30 days in soil.

As mentioned in the above results, this issue provides the recent development of advanced materials for energy and environment applications. More importantly, we hope that more attention should be paid to the research of these fields and rapid progress will be achieved in the near future.

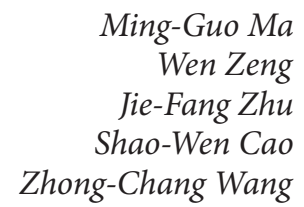



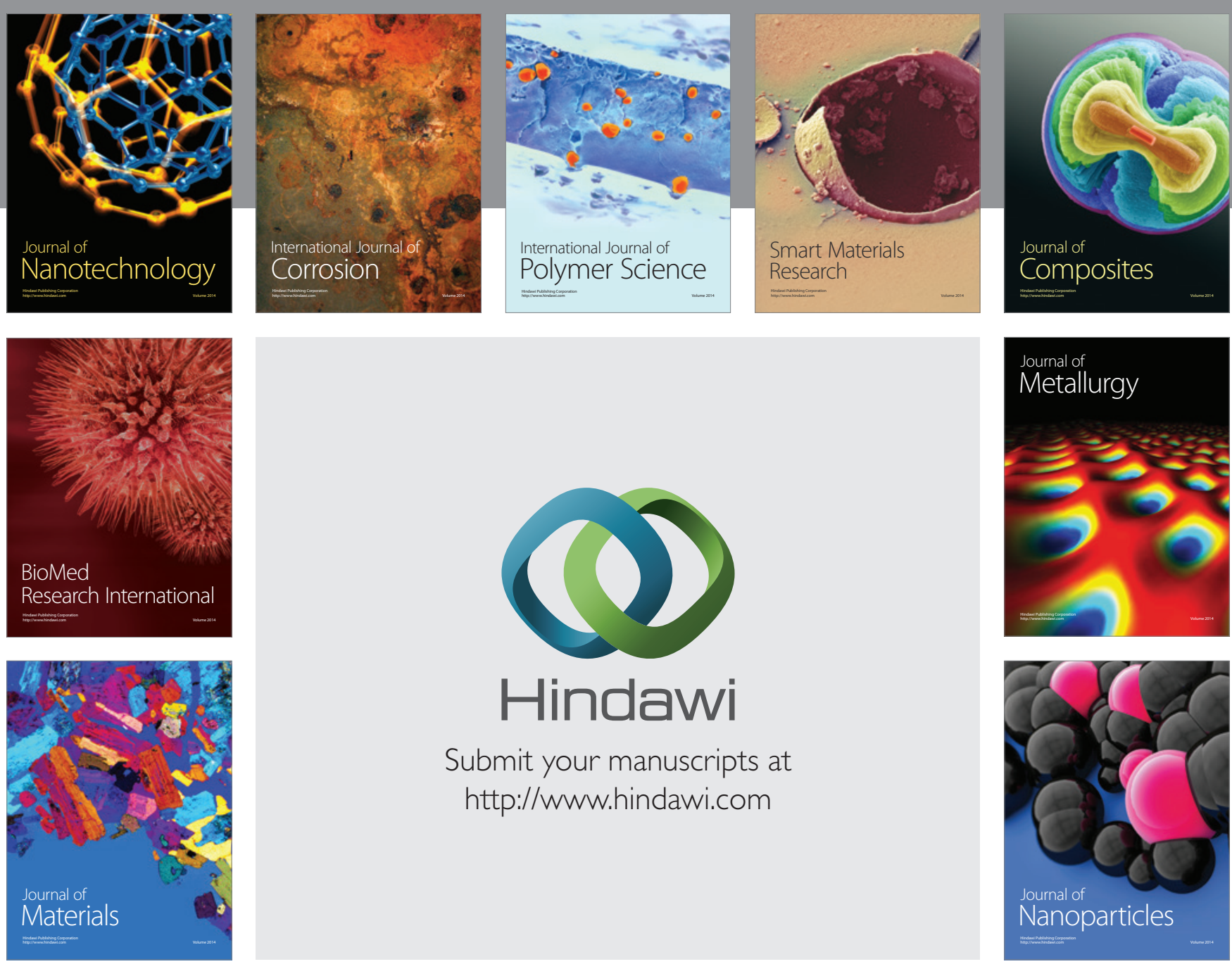

Submit your manuscripts at http://www.hindawi.com
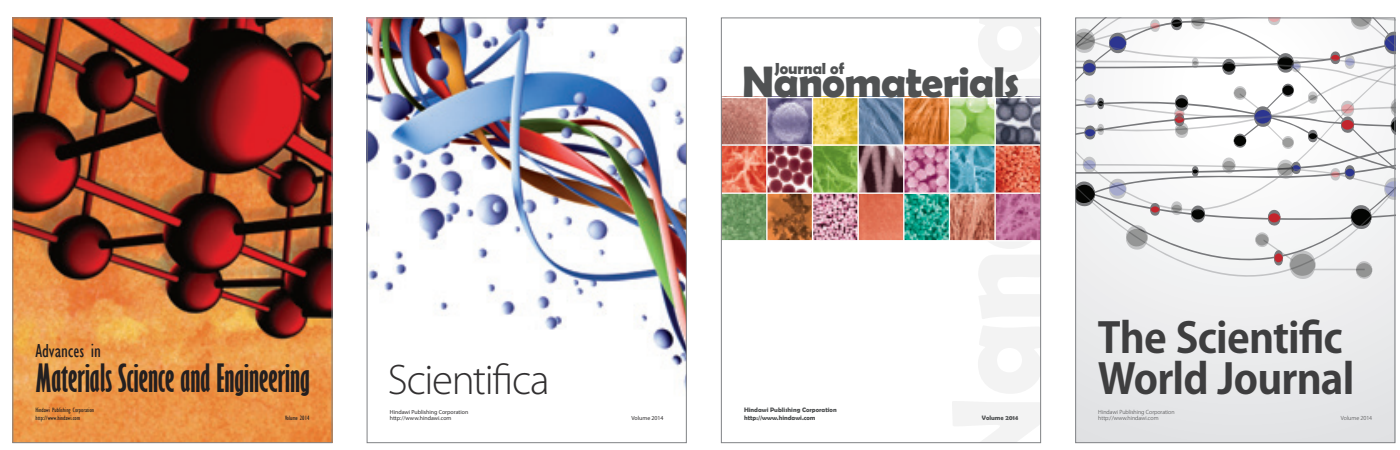

\section{The Scientific World Journal}
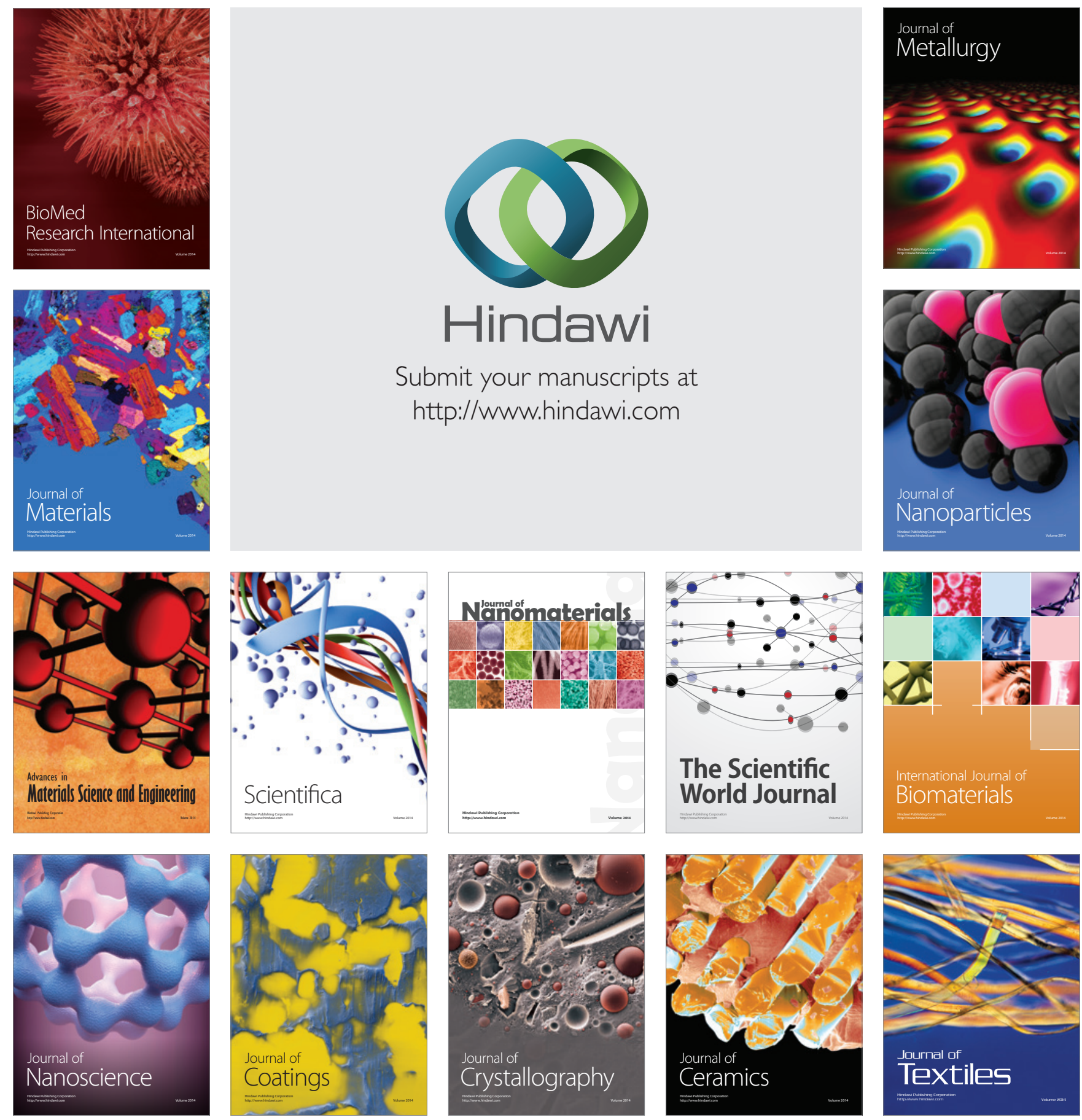